\title{
CORONAL ABUNDANCES DETERMINED FROM ENERGETIC PARTICLES
}

\author{
D. V. Reames
}

NASA Goddard Space Flight Center, Code 661, Greenbelt, MD 20771, U.S.A.

\begin{abstract}
Solar energetic particles (SEPs) provide a measurement of coronal element abundances that is highly independent of the ionization states and temperature of the ions in the source plasma. The most complete measurements come from large 'gradual' events where ambient coronal plasma is swept up by the expanding shock wave from a coronal mass ejection. Particles from 'impulsive' flares have a pattern of acceleration-induced enhancements superimposed on the coronal abundances. Particles accelerated from high-speed solar wind streams at corotating shocks show a different abundance pattern corresponding to material from coronal holes. Large variations in $\mathrm{He} / \mathrm{O}$ in coronal material are seen for both gradual and impulsive-flare events but other abundance ratios, such as $\mathrm{Mg} / \mathrm{Ne}$, are remarkably constant. SEP measurements now include hundreds of events spanning 15 years of high-quality measurement.
\end{abstract}

\section{INTRODUCTION}

Energetic particles, associated with large eruptive solar events, have provided both the earliest and the most complete information on element abundances in the solar corona. The first observation of energetic heavy ions from the Sun was made over 30 years ago by Fichtel and Guss /1/ using nuclear emulsion detectors flown on sounding rockets, and these observations were extended up to the element Fe a few years later /2/. During the 1970's instruments on satellites led to substantial improvements in the both the element resolution and statistics of the measurements. In a classic review of these measurements of solar-energetic-particle (SEP) abundances, Meyer $/ 3 /$ found that the abundances, averaged over many events, showed a twolevel dependence upon the first ionization potential (FIP) of the element, indicating an ion-neutral fractionation as material is transported up to the corona. Superimposed on the average abundances were eventto-event abundance variations that were increasingly large for heavier elements. This 'mass bias' resulted from a dependence of the acceleration process on the charge to mass ratio, Q/A, that the ions have when they are accelerated from the ambient coronal plasma at a temperature of $\sim 2 \mathrm{MK} / 4,5 \%$.

As Meyer $/ 3 /$ recognized, there was also a separate class of SEP events, called ${ }^{3} \mathrm{He}$-rich events, that have $\sim 1000$-fold enhancements in ${ }^{3} \mathrm{He} /{ }^{4} \mathrm{He}$ and $\sim 10$-fold enhancements in $\mathrm{Fe} / \mathrm{O}$. In these events, large enhancements arising from wave-particle interactions during acceleration prevented an easy determination of the underlying abundances in the source plasma.

During the last several years there has been a substantial revision in our understanding of the origin and acceleration of particles at the Sun $/ 6 /$. We have found that most of the large classic 'gradual' SEP events are not associated with solar flares but, rather, with coronal mass ejections (CMEs). Particles are accelerated from the ambient plasma at the shock wave driven ahead of the CME in these events. It is the ${ }^{3} \mathrm{He}$-rich events that come from impulsive flares. In impulsive flares, intense beams of electrons generate electromagnetic ion cyclotron (EMIC) waves below the proton gyrofrequency where they are resonantly absorbed by ${ }^{3} \mathrm{He}$, enhancing its abundance in the accelerated particles by several orders of magnitude. A new understanding of the physics of acceleration has helped us decouple the effects of acceleration and access the abundances in the source plasma in both impulsive and gradual events. 
An entirely different population of energetic particles is accelerated at shock waves produced outside $1 \mathrm{AU}$ at corotating interaction regions (CIRs) where high-speed solar wind streams overtake low-speed streams. These regions are especially prominent during solar minimum when they often persist for many solar rotations. Particles accelerated from the high-speed stream show a different dependence of the abundances on FIP indicating a difference in the ion-neutral fractionation process beneath coronal holes $/ 7,8 /$.

Thus, three distinct populations of energetic particles have been identified that come from different coronal regions:

1) In the classic large gradual events, particles are accelerated by a shock wave driven by a coronal mass ejection (CME). As the shock traverses the corona and solar wind, it samples elements in a reasonably democratic fashion, on average, allowing us to determine fairly complete abundances for $\mathrm{Z}<30$. These abundances show the well-known dependence on the first ionization potential (FIP) of the element; elements with FIP $<9 \mathrm{eV}$ are enhanced by a factor of $\sim 4$ relative to $\mathrm{H}$ or $\mathrm{O}$

2) In impulsive flares, stochastic wave-particle interactions cause additional enhancements in abundances as heavy ions are accelerated from $\sim 3$ MK plasma overlying an active region. The acceleration occurs fairly deep in coronal active regions since high magnetic fields are required for wave formation. Recent work shows that the enhancements vary smoothly with $\mathrm{Q} / \mathrm{A}$ of the ions; the underlying abundances are roughly consistent with the average coronal abundances.

3) Especially at solar minimum, particles are accelerated from high-speed solar-wind streams by shocks formed at corotating interaction regions beyond the orbit of Earth where high-speed streams overtake low-speed streams. The abundances presumably reflect the abundances in coronal holes where the high-speed streams are formed. They show a reduced FIP effect of different character from that in the other events.

Energetic particle abundances have been reviewed most recently by Reames $/ 8 /$ and they have been extensively discussed in the context of other measurements by Meyer 19, 10,11\%. Additional work on abundances, especially in impulsive-flare events $/ 12,13 /$, has been completed since the time of those reviews.

\section{COMPARING ABUNDANCE MEASUREMENT TECHNIQUES}

At a conference where many techniques are discussed for deriving abundances that may refer to the same region of the Sun, it is extremely important to understand clearly the differences and the limitations of each technique. For example, it is not uncommon to hear the comment that particle abundances are measured "way out in space" while atomic line abundances are measured "in the solar atmosphere." As yet, there are no in situ measurements of solar abundances and the X-ray or EUV photons must come out near Earth to be measured just like the energetic particles.

In fact, we can make a close analogy between the emission of photons along the line of sight and the acceleration of particles along a magnetic-field line. The physics of line emission and of acceleration can each affect the respective observations so as to obscure the underlying abundances. Comparing atomic lines with the same temperature dependence can provide abundances that are independent of variations in the source region. Similarly, comparing energetic ions with the same charge-to-mass ratio, Q/A, can yield abundances that are acceleration independent. For all of the acceleration mechanisms we know, particles with the same $Q / A$ are indistinguishable; the Lorentz force depends on particle species only as $Q$ so the evolution of the particle velocity depends only on the $\mathrm{Q} / \mathrm{A}$ of the species. However the $\mathrm{Q} / \mathrm{A}$ dependence of the acceleration can vary from event to event.

Photons come along a line of sight and, where the solar atmosphere is optically thin, that line of sight crosses plasma where temperatures and densities vary by orders of magnitude. For atomic lines, emission will occur in the temperature range where the relevant ionization state is highly probable. Only when lines have the same dependence on excitation temperature will they come from the same volume of plasma so that their ratios provide abundances. For EUV lines, emitted in the transition region where the amount of emitting material varies rapidly with temperature, it is especially important to compare lines with closely similar temperature dependence /see e.g. 14/. In flares, there may be closely spaced hotter and cooler loops. A comparison of the strength of X-ray lines emitted primarily in the hotter loops with lines that emphasize the cooler loops could depend more on the relative loop volumes than on abundances. Such effects can cause artificial 'abundance' variations from one measurement to the next. 
Energetic particles follow a particular magnetic field line that connects the source to the observer, rather than the line of sight. The interplanetary magnetic field is drawn out by the solar wind and a field line near Earth usually has a coronal footpoint near $\sim 55^{\circ} \mathrm{W}$ longitude. The spread of this particle 'field of view' is poorly known, but perhaps it is $5^{\circ}-10^{\circ}$. Particles accelerated in a flare near the footpoint will propagate to Earth in a time from $\sim 10 \mathrm{~min}$ to hours, depending on velocity, producing a ${ }^{3} \mathrm{He}$-rich event. In a gradual event, particles are accelerated from the local plasma where the shock crosses the observer's field line. For quiescent conditions, the solar wind plasma along a given field line has emerged from the same point at the top of the corona, although the plasma near Earth emerged $\sim 4$ days prior to its arrival at $1 \mathrm{AU}$. Thus energetic-particle and solar-wind abundances share a common spatial origin but differ in the time of the sample. Only one spot on the Sun is viewed at a time; this spot is swept eastward across the disk as the observer moves to new field lines because of solar rotation.

Gamma-ray lines are nuclear in origin; thus abundances derived from them do not depend on plasma temperature or density. Broad lines, emitted from excited particles of the energetic beam, suggest abundances similar to the impulsive-flare particles seen at $1 \mathrm{AU} / 15 /$. Narrow lines are produced by nuclei of the ambient plasma that are struck as the beam penetrates deep into the chromosphere and photosphere near the footpoints of the loop fields.

Particle acceleration can only occur in a low-density ionized plasma that is essentially collisionless. Acceleration is unlikely at densities above $10^{10} \mathrm{~cm}^{-3}$. The SEP spectra, abundances, and ionization states are sensitive to traversal of material that will cause energy loss, fragmentation, and changes in charge. Observations show little evidence that the particles have traversed material during or after acceleration. Ionization states of the energetic particles $/ 4 /$ (e.g. Fe ${ }^{+14}$ in gradual and $\mathrm{Fe}^{+20}$ in impulsive events) suggest that the source plasma has a temperature at or above $\sim 2 \mathrm{MK}$ in all cases.

\section{SEP MEASUREMENTS IN LARGE GRADUAL EVENTS}

Energetic particle abundances are most commonly measured in Si solid state detectors using the $\mathrm{dE} / \mathrm{dx}$ vs. E technique. The behavior of $\mathrm{dE} / \mathrm{dx}$ vs. $\mathrm{E}$ is described by the well-known Bethe-Block formula. The energy loss rate varies as $Z^{2}$ at high energies. Its integral leads to the range-energy relation which is tabulated for a wide range of stopping materials $/ 16,17 /$. For energetic-particle detectors, the energy loss rate is measured in one or more thin detectors and the residual energy is measured in a thicker detector where the particle stops. The Si detectors operate as reverse biased diodes that collect the electron-hole pairs producing a pulse height proportional to the energy deposit. All information on the initial ionization state of the particle is lost when the particle attains equilibrium charge immediately after it enters the front detector.

A density plot of pulse heights from one of the VLET telescopes on ISEE 3 is shown in Figure 1. Each point represents one particle and the observations have been summed over 43 large events. Figure 2 shows a histogram of the atomic number deduced, for particles in the 5-12 $\mathrm{MeV} / \mathrm{amu}$ interval, by superposing calibration (range-energy) curves on measurements like those in Figure 1. There are now particle telescopes with much higher resolution than the

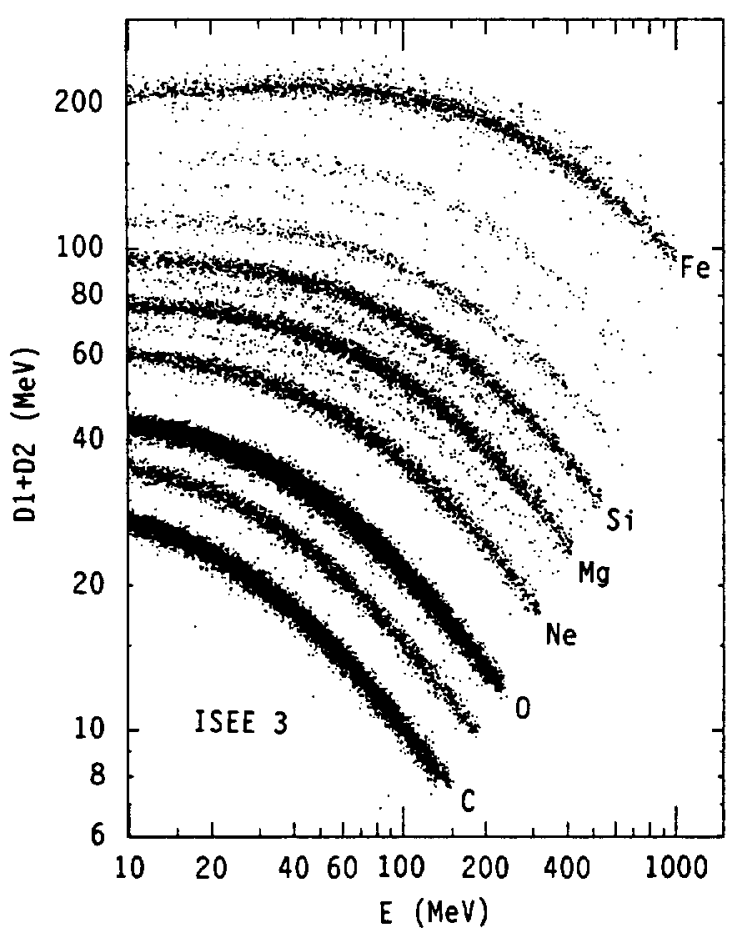

Fig. 1. A density plot showing the response of an ISEE 3 VLET telescope to ions from $\mathrm{C}$ to $\mathrm{Zn}$ accumulated over 43 large gradual events. Each point represents one particle. The measurement is completely insensitive to the ionization state of the elements. 


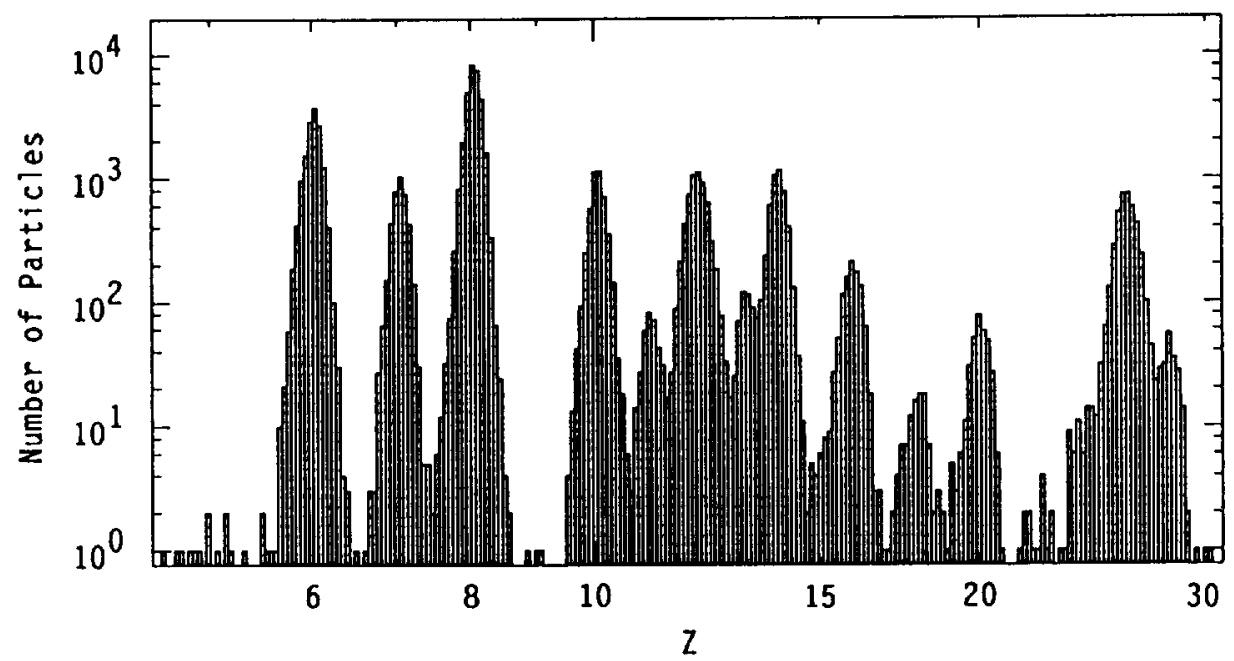

Fig. 2. A histogram of the number of particles $v s . Z$ in the 5-12 MeV/amu interval from Fig. 1.

ISEE 3 VLET (designed nearly 20 years ago), in fact with isotope resolution, but they operate at higher energies where intensities are lower and the effects of acceleration are more pronounced $/ 18 \%$. From the density of points in Figure 1 or the peak amplitudes in Figure 2, it is already clear that the abundances of $\mathrm{Ne}, \mathrm{Mg}$ and $\mathrm{Si}$ are comparable, for example, a characteristic we now ascribe to be coronal abundances.

The average element abundances in large gradual events, derived from the measurements described above, are given in Table 1. Mazur /18/ has shown that acceleration effects and event-to-event variations are minimum at low energies $\sim 1 \mathrm{MeV} / \mathrm{amu}$, especially for $\mathrm{H} / \mathrm{He}$. Therefore, the $\mathrm{H} / \mathrm{He}$ abundance is derived from 1-4 MeV/amu measurements. Heavier element abundances are derived in the 5-12 MeV/amu region where the resolution is somewhat improved. These abundances do not differ appreciably from those quoted previously /8/ where abundances of the dominant elements were measured in 2-3 MeV/amu interval. The most extreme case, $\mathrm{Fe} / \mathrm{O}$, decreases by $\sim 10 \%$ in going from the $2-3$ to the $5-12 \mathrm{MeV} / \mathrm{amu}$; this is within the uncertainty determined from event-to-event variations discussed in the next section. While the SEP abundances in Table 1 represent a complete redetermination based on new measurements, they do not differ

TABLE 1. Energetic-Particle Abundances Measured on ISEE 3

Z FIP Photosphere $\begin{gathered}\text { SEP Corona } \\ \text { (Gradual Events) }\end{gathered} \begin{aligned} & \text { Coronal Holes } \\ & \text { (CIR Events) }\end{aligned}$ ( ${ }^{3}$ He-rich Events)

\begin{tabular}{|c|c|c|c|c|c|c|}
\hline $\begin{array}{l}\mathrm{H} \\
\mathrm{He} \\
\mathrm{C} \\
\mathrm{N} \\
0 \\
\mathrm{~F} \\
\mathrm{Ne} \\
\mathrm{Na} \\
\mathrm{Mg} \\
\mathrm{Al} \\
\mathrm{Si} \\
\mathrm{P} \\
\mathrm{S} \\
\mathrm{Cl} \\
\mathrm{Ar} \\
\mathrm{K} \\
\mathrm{Ca} \\
\mathrm{Ti} \\
\mathrm{Cr} \\
\mathrm{Fe} \\
\mathrm{Ni} \\
\mathrm{Zn}\end{array}$ & $\begin{array}{r}1 \\
2 \\
6 \\
7 \\
8 \\
9 \\
10 \\
11 \\
12 \\
13 \\
14 \\
15 \\
16 \\
17 \\
18 \\
19 \\
20 \\
22 \\
24 \\
26 \\
28 \\
30\end{array}$ & $\begin{array}{l}13.53 \\
24.46 \\
11.22 \\
14.48 \\
13.55 \\
17.34 \\
21.47 \\
5.12 \\
7.61 \\
5.96 \\
8.12 \\
10.9 \\
10.3 \\
12.95 \\
15.68 \\
4.32 \\
6.09 \\
6.81 \\
6.74 \\
7.83 \\
7.61 \\
9.36\end{array}$ & $\begin{array}{c}1.18 \times 10^{6} \\
1.15 \times 10^{5} \\
468 \\
118 \\
1000 \\
0.0351 \\
161 \\
2.39 \\
44.6 \\
3.54 \\
41.7 \\
0.433 \\
20.4 \\
0.218 \\
4.21 \\
0.157 \\
2.55 \\
0.10 \\
0.563 \\
37.9 \\
2.05 \\
0.0525\end{array}$ & $\begin{array}{rl}(1.57 \pm 0.22) \times 10^{6} \\
57000 \pm & \pm 000 \\
465 & \pm 9 \\
124 & \pm 3 \\
1000 & \pm 10 \\
150.1 & \\
152 & \pm \\
10.4 & \pm 1.1 \\
196 & \pm \\
15.7 \pm & 1.6 \\
152 & \pm \\
0.65 \pm & 0.17 \\
31.8 & \pm 0.7 \\
0.24 \pm 0.1 & 0.1 \\
3.3 & \pm 0.2 \\
0.55 \pm & 0.15 \\
10.6 \pm & 0.4 \\
0.34 \pm 0.1 & 0.1 \\
2.1 & \pm 0.3 \\
134 & \pm 4 \\
6.4 \pm & 0.6 \\
0.11 \pm & 0.04\end{array}$ & $\begin{array}{r}(1.81 \pm 0.24) \times 10^{6} \\
159000 \pm 10000 \\
890 \pm 36 \\
140 \pm 14 \\
1000 \pm 37 \\
170 \pm 16 \\
140 \pm 14 \\
100 \pm 12 \\
50 \pm\end{array}$ & 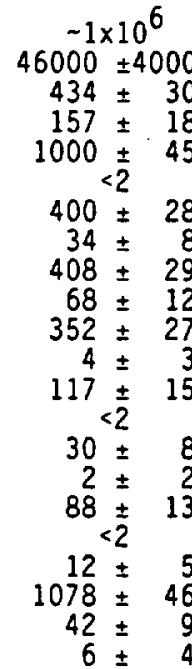 \\
\hline
\end{tabular}




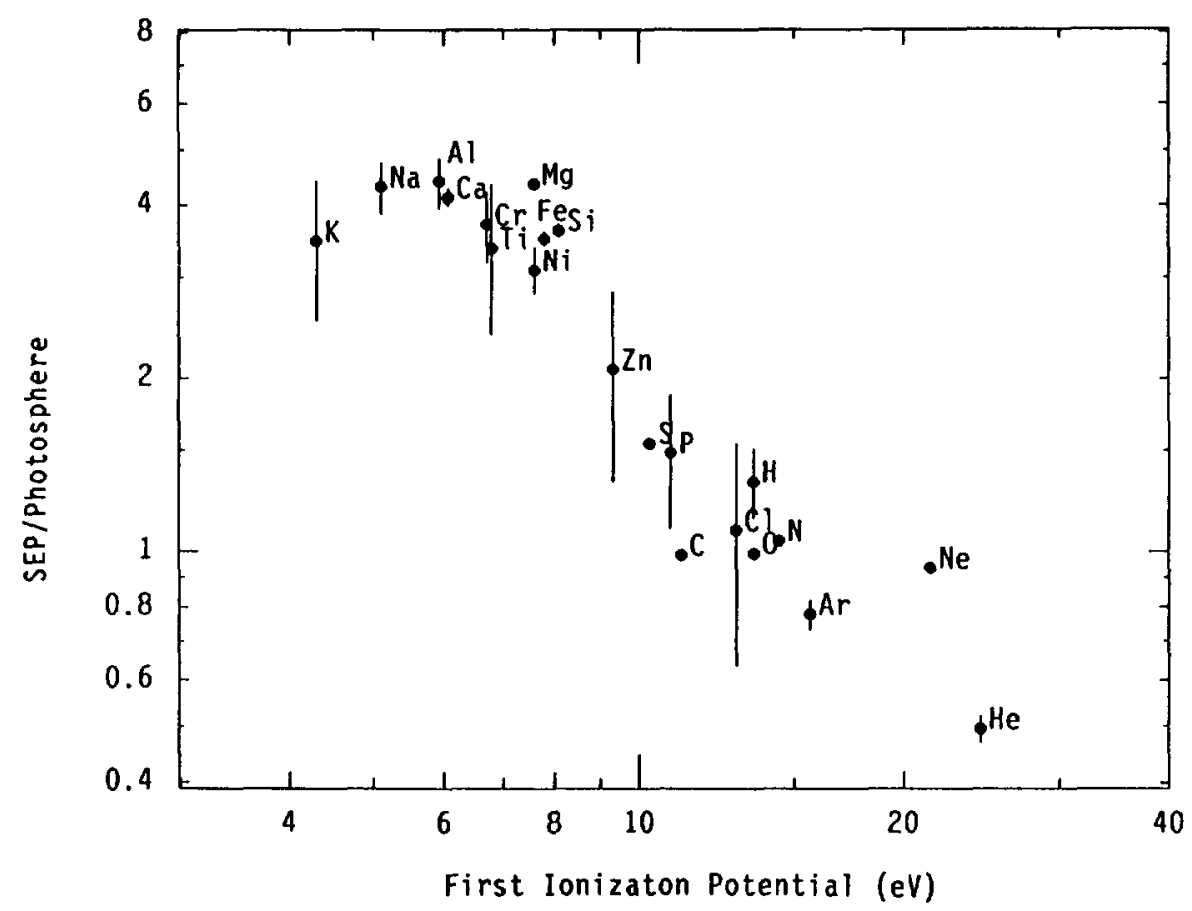

Fig. 3. Ratio of the mean abundances of elements in gradual SEP events to corresponding photospheric abundances $v s$. the first ionization potential (FIP) of each element.

significantly from previous SEP abundances $/ 8$, 19/. The $\mathrm{H}$ abundance has increased by $30 \%$ compared with the earlier measurement $/ 8 /$ because we now weight flares equally; the previous weighting by statistical errors seemed inappropriate since the scatter of the measurements far exceeds the statistical errors, as discussed in the next section.

The ratio of SEP/photospheric abundances is shown as a function of FIP in Figure 3. The photospheric abundances are from Anders and Grevesse /20/ updated for recent information on C, $\mathrm{N}, \mathrm{Ne}$ and $\mathrm{Fe} /$ see 12/. Note that abundances of $\mathrm{He}, \mathrm{Ne}$ and $\mathrm{Ar}$ are not directly measured in the photosphere.

\section{SEP ABUNDANCE VARIATIONS IN GRADUAL EVENTS}

Meyer /3/ noted that the SEP abundances showed increasingly large event-to-event variations at increasingly higher $Z$. This 'mass bias' resulted from changes in the $Q / A$ dependence of the acceleration in different events. Measurements of Q/A of the particles /4/ show that it varies systematically with $Z$ from 0.475 for $C$ to 0.266 for $\mathrm{Fe}$. Breneman and Stone $/ 19 /$ fit the abundances for $\mathrm{Z}>6$ using a power law as a function of $\mathrm{Q} / \mathrm{A}$ where the power varied from event to event. A plot of the abundances $v s$. the measured values of $\mathrm{Q} / \mathrm{A}$ is shown for 2 extreme sample events in Figure 4.

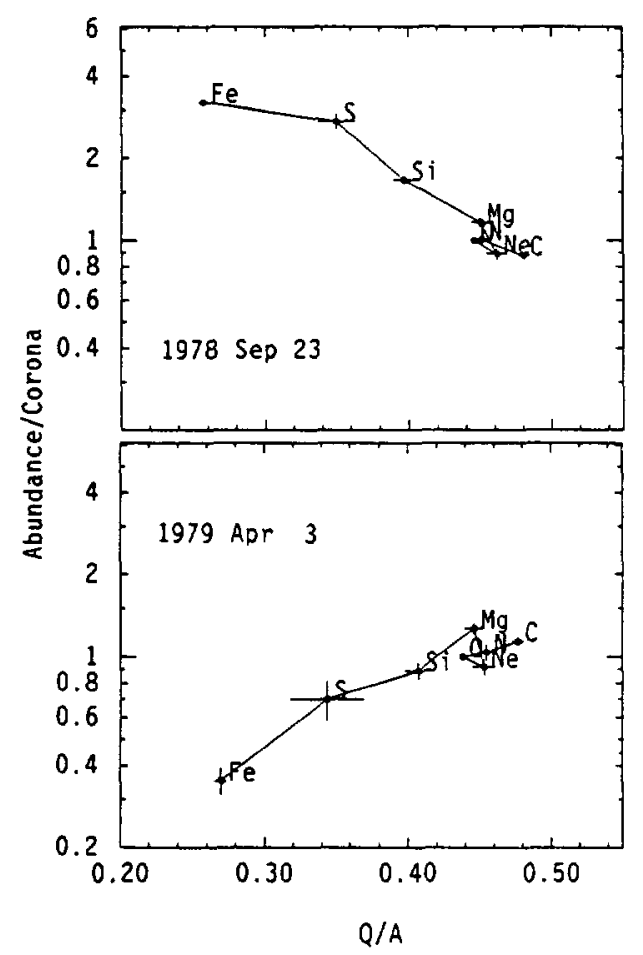

Fig. 4. The $\mathrm{Q} / \mathrm{A}$ dependence in relative abundances for 2 extreme events shows the correlated effect of acceleration /19/. Since the elements $\mathrm{C}-\mathrm{Mg}$ have similar (measured /4/) values of $\mathrm{Q} / \mathrm{A}$ in all events, their abundances are highly independent of acceleration. 
Unfortunately, Breneman \& Stone used an incorrect value for the photospheric abundance of Fe, making the $\mathrm{Q} / \mathrm{A}$ dependence seem much larger than it actually is. This means that the large correction that Breneman and Stone applied to convert from the average SEP to coronal abundances is not correct. When the meteoritic abundance of $\mathrm{Fe}$ is used for the photosphere/20/, the average SEP abundances are almost identical to the coronal abundances, and no correction is required. Furthermore, Breneman and Stone did not include $\mathrm{H}$ or $\mathrm{He}$ in their analysis. We will see that variations in both these species are uncorrelated with $\mathrm{Fe} / \mathrm{O}$, so the power-law description cannot extend to $\mathrm{Q} / \mathrm{A}=1$. Mazur $/ 18 /$ fit the energy spectra of $\mathrm{H}, \mathrm{He}, \mathrm{O}$ and $\mathrm{Fe}$ in 10 large SEP events using several theoretical acceleration models. Not surprisingly, the form of the Q/A dependence changes with energy. For all cases, Mazur found that, toward lower energy, the eventto-event variations in the abundances diminish and the abundances themselves approach coronal values.

In our present understanding of diffusive shock acceleration $/ 21 /$, particles receive an increment in velocity each time they cross the shock as they are scattered back and forth by self-generated waves. The resonance scattering between particles and waves depends upon the gyrofrequency of the particle, hence upon Q/A. Leakage of the particles from the acceleration region, dependent upon the scattering, will be small at low energies, but will increase at high energies where the intensity of both particles and resonant waves diminishes. Thus it is not surprising that abundances at low energies reflect those of the source material while those at high energies vary widely depending upon both the Q/A of the species and the properties of the shock.

Given this understanding, we examine the low-energy event-to-event variations for the 49 event periods used previously to determine the average abundances. Figure 5 shows the distributions for several abundance ratios in the events. For the elements from $\mathrm{C}$ through $\mathrm{Si}$, the standard deviation is within $20 \%$ of the mean. Thus any single event would show a FIP dependence that is very similar to that of the mean shown in Figure 3. The error in the mean for these elements is less than $-3 \%$. For $\mathrm{H} / \mathrm{He}, \mathrm{He} / \mathrm{O}$ and $\mathrm{Fe} / \mathrm{O}$, the variations are much larger. We show these ratios and $\mathrm{Si} / \mathrm{O}$ as a function of $\mathrm{Fe} / \mathrm{O}$ in Figure 6. Here it is clear that the variations in $\mathrm{Si} / \mathrm{O}$ are correlated with those in $\mathrm{Fe} / \mathrm{O}$; however, the variations in $\mathrm{H} / \mathrm{He}$ and $\mathrm{He} / \mathrm{O}$ are certainly not. In general, quoted errors on the SEP abundances in Table 1 include the mean event-to-event variations as well as statistical error and charge-resolution errors.

Variations in He/O shown in Figure 6 and similar variations in $\mathrm{He} / \mathrm{C}$ are the most difficult to explain. $\mathrm{He}$ and $\mathrm{C}$ have very similar values of $\mathrm{Q} / \mathrm{A}$ so one would expect differences in acceleration to be minimal. It is likely that the FIP-dependent mechanism that gives $\mathrm{He}$ an unusually low abundance in the corona also causes larger variations in that abundance.

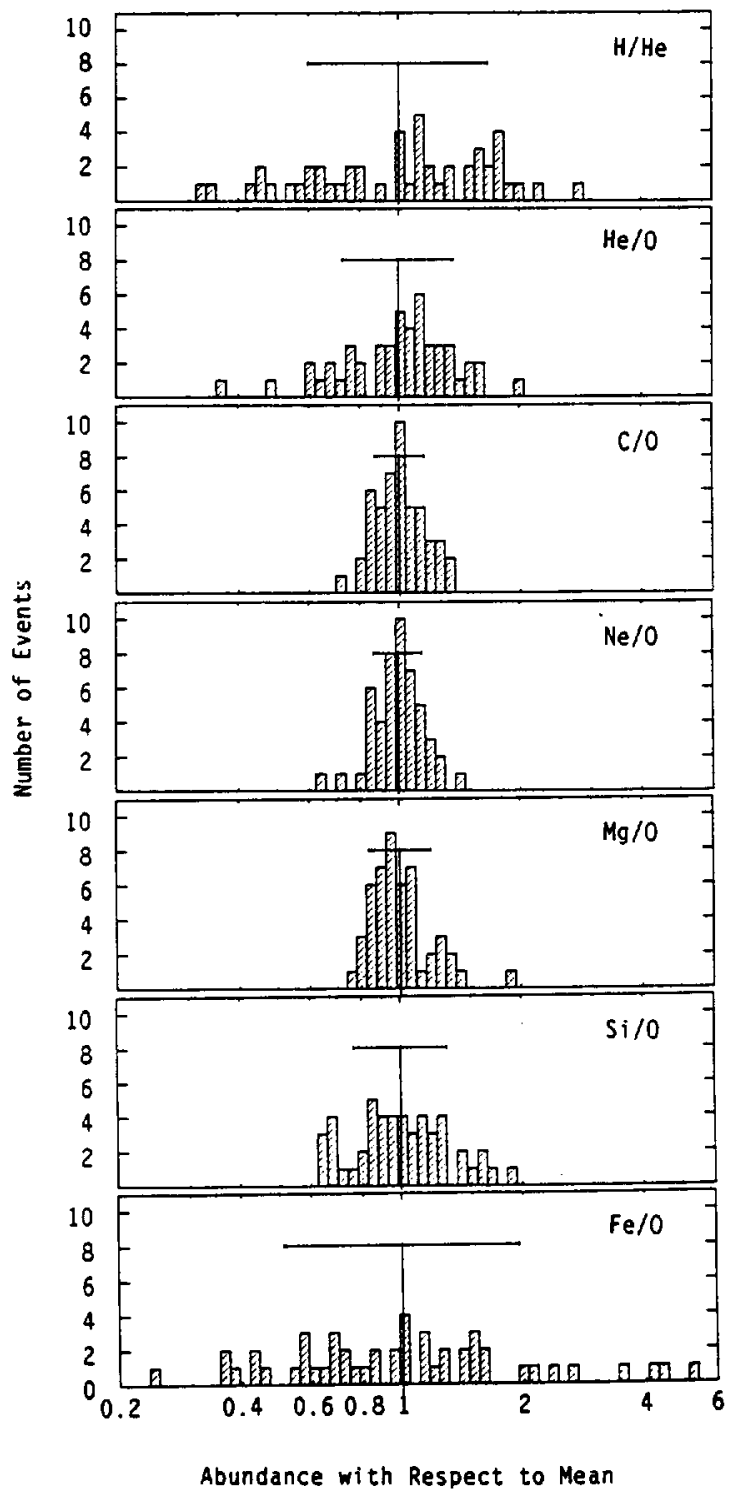

\section{IMPULSIVE-FLARE ABUNDANCES}

The large acceleration-induced enhancements of ${ }^{3} \mathrm{He}$ and heavier elements have provided fertile new

Fig. 5. Distribution of various abundance ratios in individual gradual SEP events about the mean. 


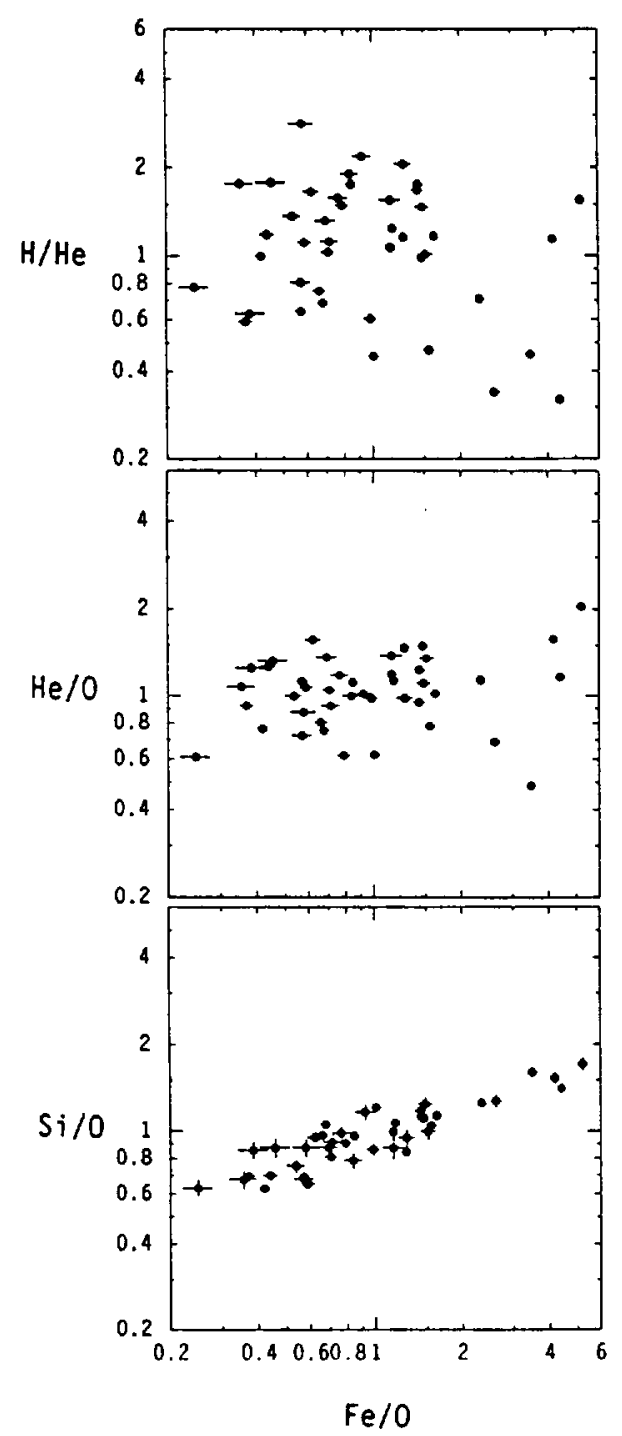

Fig. 6. For gradual events, event-to-event variations in abundances of the heavier elements correlate as shown by $\mathrm{Si} / \mathrm{O} v s$. Fe/O, indicating each event has a systematic $\mathrm{Q} / \mathrm{A}$ dependence. (at a given velocity). $\mathrm{H}$ and $\mathrm{He}$ do not fit this model.

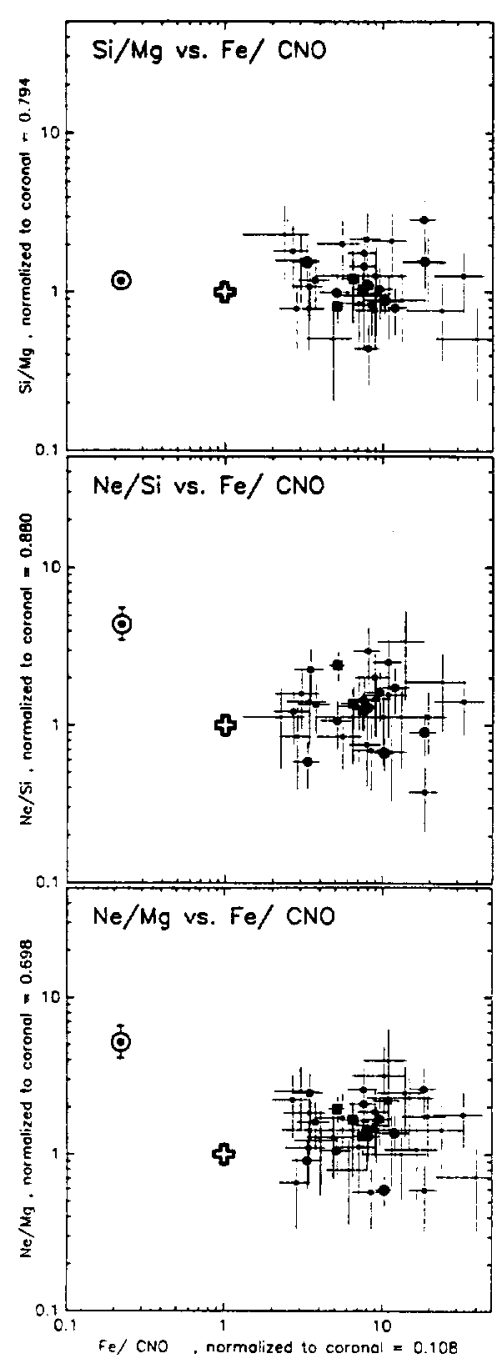

Fig. 7. In impulsive events, various ratios of the elements $\mathrm{Ne}, \mathrm{Mg}$ and $\mathrm{Si}$ are independent of $\mathrm{Fe} / \mathrm{CNO}$ and their average abundances lie much closer to the coronal (cross) than photospheric (circle) values /from $12 \%$

ground for the study of the plasma physics of wave-particle interactions in solar flares. However, to the extent that the large enhancements have stimulated studies of acceleration, they have also obstructed our view of underlying abundances in the flare plasma. Recently we have been able to better characterize the enhancements in impulsive flares based on a sample of $\sim 200$ events $/ 12 /$ and, at the same time, there has been significant improvement in the theoretical understanding of the acceleration /22-24/.

In general the pattern is one of increasing enhancement with increasing $Z$ (decreasing $Q / A$ ) with the dominant elements falling into three groups. $\mathrm{He}, \mathrm{C}, \mathrm{N}$, and $\mathrm{O}$ form the first group, with essentially coronal, unenhanced abundances. In the second group, $\mathrm{Ne}, \mathrm{Mg}$, and $\mathrm{Si}$ are each enhanced by a factor of $\sim 3$ relative to $\mathrm{CNO}$, and finally, $\mathrm{Fe}$ is enhanced by a factor of $\sim 10$ relative to CNO. Figure 7 shows the variation of three ratios of the $\mathrm{Ne}, \mathrm{Mg}$, and $\mathrm{Si}$ abundances $v s$. $\mathrm{Fe} / \mathrm{CNO}$, normalized to coronal abundances, for a sample of impulsive flare events $/ 12 \%$. The corresponding photospheric (circle) and coronal (cross) abundances are also shown. The photospheric point moves dramatically in the three panels reflecting the presence of high-FIP ( $\mathrm{Ne}$ ) and low-FIP (Mg and $\mathrm{Si}$ ) elements in the ratio, but the pattern of the impulsive- 
flare abundances remains fixed relative to the coronal point. While the Ne abundance is slightly elevated relative to $\mathrm{Mg}$ and $\mathrm{Si}$, on average, it seems likely that the energetic particles are accelerated from coronal plasma.

The grouping of the abundances in impulsive events can be understood in terms of similarities in the Q/A of the members of each group. Figure $8 / 12 /$ shows the the variation of the average value of Q/A vs. temperature based on the calculations of Arnaud and Rothenflug /25/ and Arnaud and Raymond /26/ (for $\mathrm{Fe}$ ). Note that in the vicinity of 2-5 MK, there is a plateau region where $\mathrm{Ne}, \mathrm{Mg}$ and $\mathrm{Si}$ are all in $\mathrm{He}$-like states with 2 orbital electrons so that $\mathrm{Q} / \mathrm{A} \sim 0.42$. At these temperatures $\mathrm{He}, \mathrm{C}, \mathrm{N}$, and $\mathrm{O}$ are all almost fully ionized so $\mathrm{Q} / \mathrm{A} \sim 0.5$ while $\mathrm{Fe}$ has $\mathrm{Q} / \mathrm{A} \sim 0.28$. Since ions with the same $\mathrm{Q} / \mathrm{A}$ cannot be distinguished by the acceleration mechanism, those in each group have the same enhancement and have the same relative abundances as in the source plasma. Thus the enhancement pattern is a consequence of the source plasma temperature. Now the ions are observed to have higher values of $\mathrm{Q} / \mathrm{A}$ when they are detected $/ 5 /$, thus they must be further ionized during or after acceleration. Either they are ionized by heated thermal electrons, or they are actually ionized by the same electron beams that generate the waves that cause their acceleration $/ 23 /$.

Given the plasma temperature at the beginning of the acceleration, we can calculate $\mathrm{Q} / \mathrm{A}$ for all elements and plot the mean enhancement in impulsive relative to gradual events (which represent mean coronal abundances). Figure $9 / 13 /$ shows a fairly smooth dependence of the enhancement $v s$. Q/A at 3.2 MK, thus the impulsive-flare abundances are consistent with a coronal-abundance source and smooth acceleration-induced enhancement.

For some elements there are large non-statistical variations in abundances from one event to another in the impulsive flares and these variations are not well correlated with those of other elements. The ${ }^{4} \mathrm{He} / \mathrm{C}$ ratio in a single event deviates from the mean by a factor of 1.8 , for example, and is 2.5 times the statistical variance. Since both both ${ }^{4} \mathrm{He}$ and $\mathrm{C}$ have $\mathrm{Q} / \mathrm{A} \sim 0.5$, this abundance variation must be a property of the underlying plasma, not the acceleration. Thus the impulsive events confirm the large variations in coronal He seen in the gradual events.

The elements $\mathrm{Ne}, \mathrm{Mg}, \mathrm{Si}$, and $\mathrm{Fe}$ also show large non-statistical variations that can be seen in Figure 7. In this case, however, we cannot rule out changes in the acceleration dependence on $\mathrm{Q} / \mathrm{A}$, or temperature-induced changes in $\mathrm{Q} / \mathrm{A}$ itself in different events. In addition, the abundance of $\mathrm{Ne}$ is also about $50 \%$ higher than that of $\mathrm{Mg}$ or $\mathrm{Si}$ in impulsive events. This value and its large variance supports the evidence for a higher $\mathrm{Ne}$ abundance in flares seen in gamma-ray lines $127,15 /$ and in Xray lines in some flares $/ 28 /$

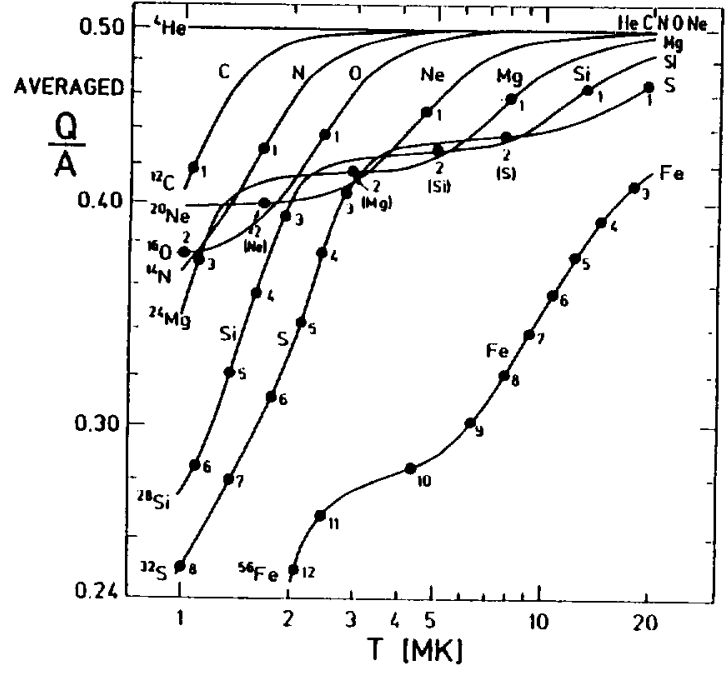

Fig. 8. Variation in the average $\mathrm{Q} / \mathrm{A}$ of dominant ions vs. temperature /from 12/ based on data from Arnaud \& Rothenflug $/ 25 /$ and Arnaud \& Raymond $126 \%$. Actual ionization states with integral numbers of orbital electrons are indicated. Note the plateau region near $\sim 3 \mathrm{MK}$ where $\mathrm{Ne}, \mathrm{Mg}$ and $\mathrm{Si}$ each have 2 orbital electrons and similar values of $\mathrm{Q} / \mathrm{A}$ while $\mathrm{He}, \mathrm{C}$, $\mathrm{N}$ and $\mathrm{O}$ are fully stripped. Ions with the same Q/A must be accelerated equally.

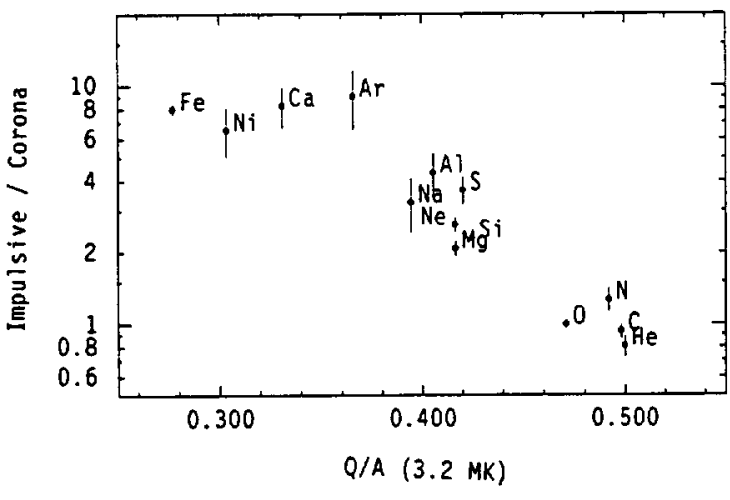

Fig. 9. Average enhancement of ions in impulsive flares relative to gradual-event (coronal) abundances vs. the mean $\mathrm{Q} / \mathrm{A}$ of the ions at 3.2 MK /from 13/. 


\section{CIR ABUNDANCES}

The final solar abundance sample provided by energetic particles comes from corotating interaction regions (CIRs) where high-speed solar wind streams overtake low-speed flows. A pair of shocks are formed at CIRs, the forward shock propagates outward into the low-speed streams and the reverse shock propagates inward through the high-speed stream. The CIR shocks generally form outside $1 \mathrm{AU}$ and particles accelerated from the high-speed stream at the reverse shock can be observed streaming sunward in the plasma rest frame. The abundances of these particles are of particular interest since they represent abundances of material emerging from coronal holes. Since the particles are shock accelerated like those in gradual events, we might expect that the abundances of the low-energy particles, averaged over many events, would again yield the abundances in the source plasma.

The observed abundances in CIRs /7, 8, 29/ are listed in Table 1 and the CIR/Photospheric abundance ratios are plotted as a function of FIP in Figure 10. The FIP dependence is more modest for the material emerging from coronal holes than for that in the corona above active regions. An examination of CIR abundances as a function of the maximum speed of the high-speed stream $/ 29 /$ shows that the abundances vary smoothly between those of gradual events (Figure 3) and those of the highspeed CIRs (Figure 10) as the stream speed increases.

It is important to distinguish these high-speed streams from coronal holes from the high-speed material ejected in the CMEs that produce gradual events. In the latter case, the shock is driven through the corona or the low-speed solar wind ahead of the CME. Since CMEs rarely form a reverse shock, the fast material in and behind the CME is not accelerated, so we cannot observe it at all. The reverse shock from the CIR propagates into the high-speed stream, sampling and accelerating particles from that population. In principle it would be possible to see particles from the forward CIR shock on field lines in the lowspeed wind, but in practice these particles are far fewer in number and more difficult to measure.

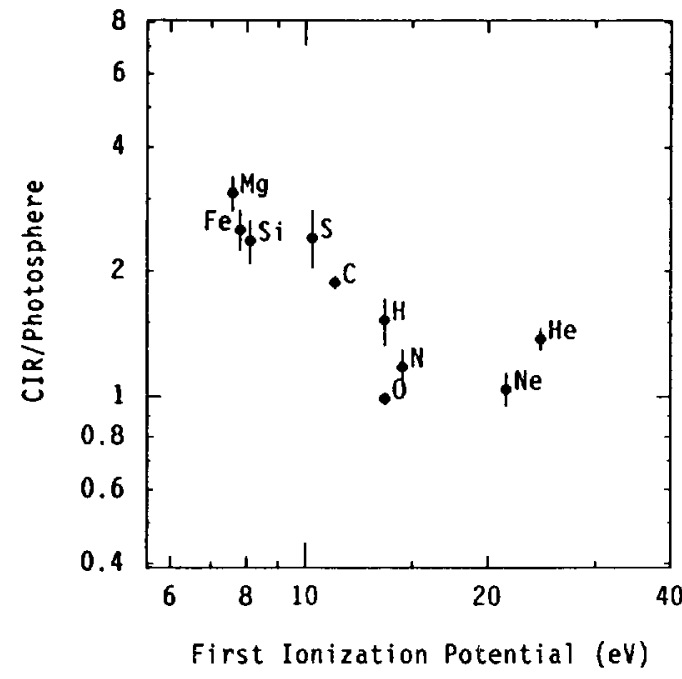

Fig. 10. The average abundances of elements accelerated from high-speed solar-wind streams at CIRs relative to photospheric abundances vs. FIP. The abundances show a reduced FIP effect for the elements emerging from coronal holes.

\section{CONCLUSIONS}

The measurement of SEP abundances is completely independent of ionization state while the acceleration depends only weakly on Q/A. Recent improvements in our understanding of particle acceleration mechanisms has helped us distinguish the effects of acceleration and deduce the element abundances in the source plasma. Thus the SEP measurements provide one of the most robust, temperature-insensitive determinations of the average coronal abundances.

In gradual events the particles are accelerated from material on the observer's field line by the shock wave ahead of an expanding CME. The acceleration depends weakly upon $\mathrm{Q} / \mathrm{A}$ of the ions but the dependence changes from event to event, depending on the Alfven wave spectrum near the shock and probably also on the Mach number of the shock, the angle between the field and the shock normal, etc. These variations are smallest at low energies $/ 18$. The elements $\mathrm{C}, \mathrm{N}, \mathrm{O}, \mathrm{Ne}, \mathrm{Na}, \mathrm{Mg}, \mathrm{Al}$, and $\mathrm{Si}$, have similar values of $\mathrm{Q} / \mathrm{A}$ and their abundance pattern changes little from event to event. For example, in 43 events spanning 12 years, the strongly FIP-dependent ratio, $\mathrm{Mg} / \mathrm{Ne}$, shows a variance of only $18 \%$ with an error in the mean value of only $3 \% / 8 /$. Despite larger variations, the mean abundances of heavier low-FIP elements, $\mathrm{Ca}, \mathrm{Ti}$, $\mathrm{Cr}, \mathrm{Fe}$, and $\mathrm{Ni}$ agree reasonably well those of $\mathrm{Mg}$ and $\mathrm{Si}$ in Figure 3 . However, one could argue that the coronal abundances of these heavy elements might be $\sim 10 \%$ higher as the lower-energy measurements of these elements suggest $/ 8 /$. 
The pattern of abundances in the high-FIP region in Figure 3 is somewhat deceptive. Partially because the abundances with the largest errors are the most visible, the high-FIP abundances seem to decrease almost continuously from $\mathrm{Mg}$ to $\mathrm{He}$. The very accurate abundances of $\mathrm{C}, \mathrm{O}$ and $\mathrm{Ne}$ prevent this interpretation and form the high-FIP plateau. For Ne, of course, the photospheric abundance is not directly measured, and a two-fold increase in photospheric Ne has previously been suggested based on gamma-ray and impulsiveflare SEP abundances $/ 27,15 /$ and flare X-ray data $/ 28 /$. However, Meyer $/ 9,10,11 /$ argues convincingly from EUV and $\mathrm{X}$-ray data that $\mathrm{Ne} / \mathrm{O}=0.16$ in the photosphere, with the larger values arising from special mechanisms in flares. The photospheric $\mathrm{C} / \mathrm{O}$ ratio is well measured at $0.47 \pm 0.10 / 30 /$.

In light of the huge enhancement of ${ }^{3} \mathrm{He} /{ }^{4} \mathrm{He}$ in impulsive-flare events, it may seem surprising to suggest that the element abundances are free from other sharp resonances and have modest enhancements that depend smoothly on Q/A. However, we now understand that the ${ }^{3} \mathrm{He}$ occupies a unique position as the only species with a value of magnetic gyrofrequency $(Q / A)$ between that of the two most abundant species, $\mathrm{H}$ and ${ }^{4} \mathrm{He}$. It is now fairly clear $/ 23,24 /$ that there are no sharp resonances below the ${ }^{4} \mathrm{He}$ gyrofrequency, and the abundances support this view. There is now a self-consistent picture in which the ions in impulsive flares are accelerated from a $\sim 3$ MK pre-flare coronal plasma where the mean abundances are similar to those found in the gradual events.

Abundance measurements of ions from the high-speed stream in CIRs suggest that the FIP effect is weaker in coronal holes, as has also been observed in more direct measurements of the solar wind $/ 31 /$. The abundances are also characterized by a high value of $\mathrm{C} / \mathrm{O}=0.9$ (vs. 0.47 in the photosphere). This makes $\mathrm{C}$ behave as a low-FIP element and raises the effective energy of transition to the high-FIP plateau. The pattern of the abundances also varies with the maximum speed of the high-speed stream $/ 29,8 /$.

There is some possibility that the $\mathrm{H}$ and He abundances in CIR events are increased somewhat by the presence of interstellar pickup ions. High-FIP interstellar neutral atoms penetrate into the inner heliosphere where they are photoionized, attaining velocities up to twice that of the solar wind. These high-velocity, singly-charged ions are more likely to be accelerated at shocks; their acceleration at the shock at the heliopause results in the 'anomalous component' of energetic high-FIP ions. Observation of radially-increasing intensities of interstellar pickup ions in the solar wind inside $5 \mathrm{AU} / 32 /$ raises the possibility that they could be accelerated at CIRs. However, the low abundance of $\mathrm{O}$ (high $\mathrm{C} / \mathrm{O}$ ) strongly argues that pickup $\mathrm{O}$ cannot contribute significantly to the CIR abundance. Nevertheless, there is some possibility of interstellar contamination of $\mathrm{H}$, which can be ionized by charge exchange, and of $\mathrm{He}$, with its very high FIP.

It is important to realize that the SEP abundances in a single gradual event represent an average of material sampled from a swath of corona roughly $\sim 10^{\circ}$ in latitude and $\sim 25^{\circ}$ in longitude. For CIRs, abundances are averaged over the entire high-speed stream. Thus these measurements represent such a large-scale average that they are not necessarily in conflict with more localized measurements that show larger variations in abundance.

The most difficult SEP element abundance to determine is that of $\mathrm{H}$. The energy dependence in the observed $\mathrm{H} / \mathrm{He}$ ratios is larger than in $\mathrm{Fe} / \mathrm{O}$, for example, and the determination rests on the evidence for the convergence of the ratios at low energies for all species and events $/ 8,16 \%$. The value of $\mathrm{H} / \mathrm{He}$ is rather sensitive to event variations and to the manner in which they are averaged; this could lead to errors in the mean of $\sim 20 \%$. It is possible that the convergence of the ratios (saturation of the resonant wave spectrum) occurs at energies below $1 \mathrm{MeV} / \mathrm{amu}$. This would lead to a lower value of $\mathrm{H} / \mathrm{He}$ and better agreement between $\mathrm{H}$ and $\mathrm{O}$ on the FIP plots for both gradual and CIR events (Figures 3 and 10).

Both the gradual and impulsive events show large variations in ${ }^{4} \mathrm{He}$, as measured by ${ }^{4} \mathrm{He} / \mathrm{C}$. Since the $\mathrm{Q} / \mathrm{A}$ values of ${ }^{4} \mathrm{He}$ and $\mathrm{C}$ are similar, especially in impulsive events, we can only conclude that there are large variations in the He abundance in ambient coronal material. Thus, not only is He suppressed by a factor of 2 , on average, compared with the other high-FIP elements, but its abundance also varies by a factor of $\sim 2$, above and below the mean, from one active region to another.

I would especially like to thank J. L. R. Saba for a careful reading of this manuscript and for many helpful discussions during this work. Comments by referee, K. J. H. Phillips, were also appreciated. In addition, I have benefited greatly from discussions with J. P. Meyer, J. A. Miller and J. T. Schmelz during the course of this work. 


\section{REFERENCES}

1. C.E. Fichtel \& D.E. Guss, Phys. Rev. Letters, 6, 495 (1961).

2. D.L. Bertsch, C.E. Fichtel \& D.V. Reames, Ap. J. (Letters), 157, L53 (1969).

3. J.P. Meyer Ap. J. Suppl. 57, 151 (1985).

4. A. Luhn, B. Klecker, D. Hovestadt, G. Gloeckler, F. M. Ipavich, M. Scholer, C. Y. Fan, \& L. A. Fisk, Adv. Space Res. 4, No. 2-3, 161 (1984).

5. A. Luhn, B. Klecker, D. Hovestadt, \& E. Möbius Ap. J., 317, 951 (1987).

6. D.V. Reames, Adv. Space Res., 13, No. 9, 331 (1993).

7. D.V. Reames, I.G. Richardson \& L.M. Barbier, Ap. J. (Letters), 382, L43 (1991).

8. D. V. Reames, In: First SOHO Workshop on Coronal Streamers, Coronal Loops, and Coronal and Solar Wind Composition, Annapolis, ed. V. Domingo (ESA publ. SP-348, Paris: ESA), 315 (1992)

9. J. P. Meyer, Adv. Space Res., 11, No 1, 269 (1991).

10. J. P. Meyer, Adv. Space Res., 13, No 9, 377 (1993).

11. J. P. Meyer, In: Origin and Evolution of the Elements, eds. N. Prantzos, E. Vangioni-Flam \& M. Cassé (Cambridge Univ. Press) in press (1994).

12. D. V. Reames, J. P. Meyer \& T. T. von Rosenvinge, Ap. J. Suppl., 90, 649 (1994).

13. D. V. Reames, Proc. 23nd Internat. Cosmic Ray Conf. (Calgary), 3, 388 (1993).

14. K. G. Widing \& U. Feldman, Ap. J., 344, 1046 (1989).

15. R. J. Murphy, R. Ramaty, B. Kozlovsky \& D. V. Reames, Ap. J., 371, 793 (1991).

16. W. H. Barkas \& M. J. Berger in Studies in Penetration of Charged Particles in Matter (Nat. Acad. Sci.- Nat. Res. Council publication 1133) 103 (1964).

17. L. C. Northcliffe \& R. F. Schilling, Nuclear Data 7, 233 (New York: Academic Press) (1970).

18. J.E. Mazur, A Survey of Solar Flare Energetic Particle Abundances and Energy Spectra, PhD thesis, Univ. of Maryland PP 92-118 (1991).

19. H.H. Breneman \& E.C. Stone, Ap. J. (Letters), 299, L57 (1985).

20. E. Anders \& H. Grevesse, Geochim. Cosmochim. Acta, 63, 197 (1989).

21. M.A. Lee, J. Geophys. Res. 88, 6109 (1983).

22. M. Temerin \& I. Roth, Ap. J. (Letters), 391, L105 (1992).

23. J. Miller \& A. F. Viñas, Ap. J., 412, 386 (1993).

24. J. Miller, A. F. Viñas \& D. V. Reames, Proc. 23nd Internat. Cosmic Ray Conf. (Calgary), 3, 17 (1993), also $A p . J$. , submitted (1994).

25. M. Arnaud \& R. Rothenflug, Astron. \& Astrophys. Suppl., 60, 425 (1985).

26. M. Arnaud \& J. Raymond, Ap. J., 398, 394 (1992)

27. D. V. Reames, R. Ramaty, and T. T. von Rosenvinge, Ap. J. (Letters), 332, L87 (1988).

28. J. T. Schmelz, Ap. J., 408, 373 (1993).

29. I.G. Richardson, L.M. Barbier, D.V. Reames, \& T.T. von Rosenvinge, J. Geophys. Res., 98, 13, 1993.

30. N. Grevesse, A. Noels, \& A. J. Sauval, In: First SOHO Workshop on Coronal Streamers, Coronal Loops, and Coronal and Solar Wind Composition, Annapolis, MD, ed. V Domingo (ESA publ. SP348, Paris: ESA), 305 (1992).

31. G. Gloeckler and J. Geiss, In: Cosmic Abundances of Matter, AIP Conf. Proc. 183, ed. C. J. Waddington, (AIP: New York), 49 (1989).

32. G. Gloeckler, J. Geiss \& R. von Steiger, Adv. Space Res., to be published (1994). 